\title{
Self-Disclosure: A Psychological Analysis
}

\author{
Serhat Arslan ${ }^{1}$
}

Article History:

Received 11.11.2020

Received in revised

form 01.09.2021

Accepted

Available online

01.10 .2021

\begin{abstract}
Self-disclosure is defined as the process of expressing one's feelings and thoughts about oneself to others. It is now extensively studied by social and clinical psychologists interested in self-disclosure, interpersonal communication and close relationships. Questions about why people self-disclose have led to significant research and theory generation over the years. Little attention has been paid, however, to the potential biological underpinnings of this complex behavior. Although situational factors have a significant impact on communication patterns in close relationships, individual differences can be observed even in the face of strong social-situational stimuli. Self-disclosure is seen as a healthy personality structure and sign. It appears to be a relatively stable personality trait associated with positive personality traits. The aim of this research is to examine the concept of self-disclosure from a psychological perspective.
\end{abstract}

(C) IJERE. All rights reserved

Keywords: Self-Disclosure,disclosure, analysis

\section{INTRODUCTION}

Self-disclosure plays a central role in people's personal lives. Self-disclosure can occur in a variety of interactions or contexts, such as different relationship settings, with romantic partners and with clients, and online such as with a therapist (Nguyen et al., 2012). Disclosed personal information can include basic explicit information such as demographics (for example age, gender, height) as well as more confidential information such as attitudes and opinions, values, beliefs, feelings, needs, fantasies and fears. The information disclosed can vary in importance; the amount of information, time spent communicating, and the variety and depth of topics - some disclosure issues may be more confidential and internal than others. For example, disclosure about personal feelings, desires, thoughts, fantasies and values is considered to be at a deeper, more internal level than statements about mere facts. In the twenty-first century, many social interactions and relationships take place partially or completely online. With the many online platforms available for personal information disclosure, the high use of these platforms is driving more people online. In virtual self-disclosure, there are countless ways such as e-mails, text messages, online forums, online social networks, in-game chats and online classes (Li, 2007). Online self-disclosure does not seem to have different characteristics from offline selfdisclosure. It basically differs in the structural features of the message medium. These contextual differences can shape perceptions and responses, which include relational processes, and can yield specifically different relational outcomes (Adams et al., 2004). Self-disclosure can occur in our lives in a variety of ways, either oneon-one or as part of group counselling. Some studies have found that constructs related to online selfdisclosure are associated with negative associative outcomes.

A new relationship in everyday life often begins with the exchange of personal information. In relationships, the individual's self-disclosure ensures that the relationship is developed and reliable. If a relationship is thought to be beneficial, the individual's self-disclosure increases. The process of self-disclosure plays an important role in moving relationships from superficial to intimate. Individuals who aim to establish longterm and important relationships engage in higher levels of self-disclosure. The self-disclosure process deals with relationship uncertainty in two ways. First, the higher the level of self-disclosure in relationships, the less uncertainty there is in the relationship; Conversely, the stronger the uncertainty in a relationship, the lower the level of self-disclosure between the parties. In addition, self-disclosure reduces mutual uncertainty about thoughts and beliefs and this process significantly encourages the development of the relationship (Almakrami, 2015). West and Turner defined self-disclosure as the process of purposefully revealing information about oneself to others while revealing pieces of information about another person. When someone opens up to another person in a relationship process, this demonstrates that the recipient is loved and reliable (Tang \& Wang, 2012). Self-disclosure is seen as an indicator of the level of sincerity of expression

${ }^{1}$ Necmettin Erbakan University, serhat.arslan@erbakan.edu.tr, orcid.org/ 0000-0003-4422-8421 
and the level of trust and love in bilateral relations. Relationships can also contain a sincere quality of selfdisclosure. The recipient feels socially rewarded and feels love as a result of someone else opening up to them. In addition, in the process of self-disclosure, sincerity is defined as a deep level of communication in which the sender reveals personal information about themselves. Self-disclosure also includes common areas about the number of topics discussed in the relationship process and how much time they spend on these issues. In the process of self-disclosure, depth refers to the degree of sincerity which guides the discussion (West \& Turner, 2010).

Adler and Towne (1996) stated that self-disclosure can move from superficial communication to deeper personal information. This process is expressed as stereotypes, facts, opinions and feelings, and clichés which are polite short responses to strangers or new situations. They are also described as a reaction to ritualized social situations. Facts manifest as trust and commitment in the relationship as those who reveal facts that are generally unknown to others. Opinions include how and what a person thinks about a particular topic. Emotions are often expressed as the most obvious example of this. This process goes to the depth of the individual's views on the subject, how they recognize for themselves that the principle or the subject is true. The aim of this research is to examine the concept of self-disclosure from a psychological perspective.

\section{METHOD}

In this study, the document analysis method, which is among the qualitative research methods, was used. Content analyzes of academic studies on the concept of self-disclosure were carried out.

\section{CONCLUSION and DISCUSSION}

Self-disclosure is rewarding and provides significant benefits for individuals, but it also carries risks, especially in social media environments. Today, self-disclosure on social media platforms has become an important part of people's social life. Social media technologies greatly facilitate such sharing of personal information to a large audience through indirect status updates. Self-disclosure in social media creates challenges such as mirroring different content, increased vulnerability and a loss of privacy which limits the benefits which it can bring (Ma et al., 2016).

It has been observed that people with secure attachment are more self-disclosed, both appropriately and to a greater extent, compared with people with insecure attachment worldwide (Collins \& Read, 1990). Individuals who are less anxious in their everyday life are less emotionally dependent in their relationships. They also experience fewer ups and downs in a relationship and engage in less inappropriate self-disclosure (Pistole, 1995). The insecurities of individuals with insecure attachment in their relationships keep them away from their partners and thus lead to an unwillingness to reveal themselves or to have their partner reveal themselves. Securely attached people tend to be more self-explanatory because both attributes lead them to view others positively. The fact that the individual feels comfortable enough to reveal themselves enables them to accept that they are different in terms of the scope, expectations and reactions of the subject (Seol, 2016).

In Davis's (2012) study of online self-disclosure among adolescents, nearly half of the participants thought that it is easier to share personal feelings online. They also commented that online explanations are easier due to reduced non-verbal cues and a sense of keeping one's communication in check. Girls are more likely to self-explain online than boys. Adolescents' candid posts and statements online reflect a defining feature of adolescent friendships which play an important role in helping adolescents to give and receive feedback about their identity. Tang and Wang (2012) stated that adolescents engage in self-disclosure behavior in online environments within the scope of attitudes, body, money, work, emotions, personal interests and experiences. They also found that blog research participants were aware of the risks of far-reaching disclosure and were more willing to disclose their interests and experiences than personal and monetary issues. Among the motivations for self-disclosure behavior in adolescents, it has also been determined that users' privacy concerns and trust tendencies are important factors in whether individuals are willing to disclose personal information (Christofides et al., 2011).

There are many negative consequences which self-disclosure online can have; these can range from identity theft to the unintended disclosure of personal information. The first is a risk which is exacerbated by the widespread use of personal questions to identify a person. It has been concluded that adolescents are generally aware of the possible consequences of self-disclosure behaviors, but often do not act (significantly) on this information. It was found that their privacy settings were changed after the event in which their privacy 
was disclosed. It should be noted that experiences of online self-disclosure are not limited to unintentional disclosures, but also include "bullying, unwanted communication and misunderstandings" (Debatin, 2009). Self-disclosure is a key factor in developing relationships in online environments, as well as in face-to-face contexts. Computer-mediated environments such as social networking sites and online dating sites offer us various possibilities to initiate, develop and maintain interpersonal relationships (Dindia, 2000).

Several suggestions for future studies within the scope of this research can be made. Individuals can be informed through counselors so that they can practice self-disclosure behaviors in a healthier and safer way. Within the scope of self-disclosure problems which individuals might experience in the online environment, group counseling or individual counseling can be offered by counselors. Through the psycho-educational groups which counselors can prepare, awareness-themed studies can be conducted on the effects which users experience and might potentially experience because of psychological self-disclosure behavior.

\section{REFERENCES}

Adams, G., \& Anderson, S. L., ve Adonu, J. K. (2004). The cultural grounding of closeness and intimacy.In D. Mashek ve A. Aron (Eds.), Handbook of closeness and intimacy (pp. 321-339). Mahwah, NJ: Lawrence Erlbaum Associates.

Adler, R. B., \& Towne, N. (1996). Looking out looking in interpersonal communication (8th ed.). Cengage Learning. Almakrami,H.A. (2015). Online self-disclosure across cultures: A study of facebook use in Saudi Arabia and Australia. Bachelor of Computer Science (King Abdulaziz University), Master of IT (RMIT University).

Christofides, E., Muise, A., \& Desmarais, S. (2012). Risky disclosures on facebook: The Effect of having a bad experience on online disclosure. Journal Adolescent Research, 27(6), Doi:10.1177/0743558411432635.

Collins, N. L., \& Read, S. J. (1990). Adult attachment, working models, and relationship quality in dating couples. Journal of Personality and Social Psychology, 58, 644-653.

Davis, K. (2012). Friendship 2.0: Adolescents' experience of belonging and self-disclosure online. Journal of Adolescence, 35, 1527-1536.

Debatin, B., Lovejoy, J.P., Horn ,A,.K. \& Hughes,B.N.(2009). Facebook and online privacy: Attitudes, behaviors, and unintended consequences. Journal of Computer Mediated Communication, 15.

Dindia, K. (2000). Self-disclosure, identity, and relationship development: A dialectical perspective. In K. Dindia \& S. W. Duck (Eds.), Communication and personal relationships (pp. 147-162). Chichester, UK: Wiley.

Li, Q. (2007). New bottle but old wine: A research of cyberbullying in schools. Computers in Human Behavior, 23, 1777-1791.

Ma,X., Hancock,J. \& Naaman,M. (2016). Anonymity, intimacy and self-disclosure in social media. CHI'16, May 0712 , San Jose, CA, USA.

Nguyen, M., Bin, Y. S., \& Campbell, A. (2012). Comparing online and offline self-disclosure: A systematic review. Cyberpsychology, Behavior, and Social Networking, 15, 103-111.

Pistole. (1995). College students' ended love relationships: Attachment style and emotion. Journal of College Student Development, 1, 53-60.

Seol,J.Y. (2016). Self-disclosure in American friendships: Links with collectivism and adult attachment styles. San Francisco State University, Master of Arts.

Tang, J., \& Wang, C. (2012). Self-disclosure among bloggers: Re-examination of social penetration theory. Cyberpsychology, Behavior, and Social Networking, 15(5), 245-250.

West, R., \& Turner, L. (2010). Introducing communication theory; analysis and application (4 th Ed). New York: McGraw-Hill International Edition. 